\title{
5 Freuds Theorie zu den Mechanismen der Wahnbildung
}

\author{
Eva Horvath
}

Die Konzepte Sigmund Freuds zur Wahnbildung bzw. zu psychotischen Mechanismen sind Ausgangspunkt und Referenz für die weitere psychoanalytische Theoriebildung zu dieser Thematik, die mittlerweile in vielfältiger Differenzierung und Modifikationen von Detailaspekten vorliegt. Die Entwicklung der grundlegenden Erkenntnisse Freuds über die Paranoia soll hier im Kontext seines metapsychologischen Theoriegebäudes dargestellt werden.

\subsection{Einleitung}

Freud erklärt die bewussten und unbewussten Funktionen der Psyche und ihrer Störungen als das Resultat einer Entwicklung, die sich aus konstitutionellen Bedingungen und Umwelteinflüssen im Sinne einer Ergänzungsreihe zusammensetzt. Dabei wird dem Psychischen ein vielschichtig differenzierter Bereich mit eigenen inneren Gesetzmäßigkeiten eingeräumt, der für sich betrachtet und erforscht werden kann. ${ }^{10}$ Die Grundlage des Freud'schen Modells bildet eine dualistische Theorie der Triebe. Zunächst hatte Freud dem Sexualtrieb (der Libido) die Selbsterhaltungstriebe gegenübergestellt. Nach der im Zuge der Beschäftigung mit psychotischen Affektionen und dem Phänomen des Narzissmus gewonnenen Erkenntnis, dass die Selbsterhaltung aus der libidinösen Besetzung des Ichs entsteht und somit keinen Triebgegensatz darstellt, formulierte Freud seine zweite Triebtheorie. Libido (Eros, Sexualtrieb)

10 Der „psychophysische Parallelismus“, eine Vorstellung, die Freud von H. Jackson übernimmt und schon in seiner Studie „Zur Auffassung der Aphasien“ (1891) formuliert. 
und Destrudo (Destruktionstrieb, Todestrieb) ${ }^{11}$, zwei einander im Prinzip entgegengesetzte, im Organischen wurzelnde Triebarten, die sich miteinander in jeweils individuellen Mischungen verbinden, bilden ihre beiden Antagonisten.

Freud sieht die libidinöse Triebspannung und deren Regulation - das Verlangen nach Befriedigung und ihren Aufschub bzw. Verzicht der Triebbefriedigung unter der Berücksichtigung der Realität - als zentralen Bestandteil und Auslöser der phylo- und ontogenetischen Differenzierung des „psychischen Apparats“:

Ausgehend von Partialtrieben, die mit den frühkindlichen erogenen Zonen korrespondieren, mündet die Entwicklung der Libido in die genitale Trieborganisation, die diese sexuellen Teiltriebe in einer Zentrierung auf die Genitalorgane vereint. Aus zielgehemmten bzw. sublimierten Sexualstrebungen entstehen die Voraussetzungen für soziale Bindungen und für alle kulturellen menschlichen Leistungen. Die innerpsychischen Konflikte im kindlichen Triebleben sind sowohl Katalysator für die individuelle Entwicklung als auch Grundlage für die möglichen Störungen und Erkrankungen dieses „psychischen Apparates“, seiner Strukturen und Funktionen. Das Zentrum dieser Konflikte bilden zwei miteinander in Beziehung stehende Themen der infantilen Genitalorganisation: Kastrations- und Ödipuskomplex.

Der Kastrationskomplex beinhaltet sowohl Ängste vor der Verletzung oder dem Verlust des Genitales beim Knaben als auch den Penisneid des Mädchens - als Konsequenz der Entdeckung des Geschlechtsunterschieds - und damit zusammenhängende Phantasien über die phallische Potenz bzw. Minderwertigkeit. Der Ödipuskomplex $^{12}$ - der „Kernkomplex“ der psychoanalytischen Theorie - beschreibt die Konstellationen und Phantasien, die aus den kindlichen Liebeswünschen und Rivalitäten gegenüber dem Elternpaar resultieren.

In der kindlichen Entwicklung werden hier phylogenetische Muster (Urphantasien) in der individuellen Beziehungserfahrung zu bewussten und unbewussten Vorstellungen ausgestaltet und zu Phantasien verbunden. Diese kann man im Kontext der Triebentwicklung als symbolisierte und unbewusst komplex verbundene Darstellungen und Differenzierungen von Objektbegehren, Objektdestruktionsvorstellungen, Selbstliebe und Selbstdestruktionserleben (mit der damit verbundenen Vernichtungsangst) verstehen.

Als psychische „Instanzen“ bezeichnet Freud die Substrukturen der Psyche, deren genetische Anlage durch die individuelle Entwicklung geformt wird. Man kann diese fiktiven Strukturen als Funktionseinheiten, als „seelische Organe“" ${ }^{13}$ ansehen: das Es als Matrix, das Ich mit seinen vielfältigen Funktionen, das sich aus dem Ich differenzierende Über-Ich sowie das narzisstische Ich-Selbst-Regulationssystem.

Dieses mehrdimensionale Modell der Psyche ermöglicht es, jede psychische Äußerung bzw. Leistung, wie auch deren Funktionsstörung ausdrückende Symptombildungen wie z.B. den Wahn als komplexes Ergebnis von aktuellen und vergangenen äußeren Einflüssen, vorgegebenen und erworbenen inneren strukturellen Bedingungen und den daraus resultierenden Wechselwirkungen zu beschreiben.

11 Freud S (1920) Jenseits des Lustprinzips, SA III, S. 213-272

12 Unter „positivem Ödipuskomplex“ werden die Liebeswünsche dem gegengeschlechtlichen Elternteil gegenüber, unter „negativem Ödipuskomplex“ die Liebeswünsche dem gleichgeschlechtlichen Elternteil gegenüber verstanden.

13 Loch W (2010), S. 39 
Im Folgenden werden, ausgehend von einer kurzen Skizzierung dieser funktionalen Bereiche, die den Wahnmechanismus betreffenden Beobachtungen und Schlussfolgerungen Freuds ins Zentrum gerückt.

\subsection{Das Ich und das Es ${ }^{14}$}

Als Es bezeichnet Freud die unbewusste Matrix, den Ausgangspunkt aller strukturellen psychischen Differenzierungen. Es beinhaltet sowohl primär unbewusste als auch sekundär verdrängte Inhalte. Als regulative Instanz aus dem Es entwickelt, dient das Ich mit bewussten und unbewussten Anteilen als Vermittler zwischen den Triebansprüchen (die dem Es entstammen und ein somatisches Korrelat haben) und der Außenwelt bzw. ihren verinnerlichten Gebots- und Verbots-Einflüssen, die vom ÜberIch vertreten werden.

Für die Entwicklung der verschiedenen Ich-Leistungen spielen sowohl die Erfahrung der Befriedigung durch das Primärobjekt als auch die Unumgänglichkeit des Triebaufschubs eine strukturbildende Rolle.

Bestimmte Mechanismen des Ichs regeln analog einer Schnittstelle den Austausch zwischen den beiden Funktionssystemen Es und Ich. Inhalte des Es gelangen nicht als solche, sondern modifiziert - als Ich-kompatible Abkömmlinge - zur bewussten Wahrnehmung. Wird diese zensorische Schwelle - die sogenannte „Abwehr“ - überlastet, können Es-Inhalte als peinliche oder unerträgliche Vorstellungen und Affekte bewusst werden. Freud hat den Begriff „Abwehr“ schon in seiner Beschreibung der „Abwehr-Neuropsychosen“ 1894 verwendet, ihn dann durch „Verdrängung“ ersetzt, später dann in verschiedene Abwehrmechanismen differenziert und hinsichtlich der Paranoia typische Abwehrformationen - die Projektion und die Verleugnung - beschrieben.

Die Entstehung des Über-Ichs als besonderen Teil des Ichs erklärt Freud durch Introjektion und Identifizierung: Das Objekt wird dem Subjekt „einverleibt“, es wird „im Ich aufgerichtet“. wodurch eine Objektbesetzung durch eine Identifizierung ersetzt wird. Das Über-Ich ist zuallererst Ausdruck des Elterneinflusses, „Erbe des Ödipuskomplexes" und der langen kindlichen Hilflosigkeit und Abhängigkeit im Verein mit dem zweizeitigen Ansatz der sexuellen Entwicklung. „Als kleine Kinder haben wir diese höheren Wesen gekannt, bewundert, gefürchtet, später in uns selbst aufgenommen“" ${ }^{15}$, schreibt Freud über das Ich-Ideal oder das Über-Ich als Repräsentanzen der Elternbeziehung.

Der die Wahnbildung beherrschende Abwehrmechanismus der Projektion ist als Gegenbewegung zur Introjektion erkennbar: Etwas Störendes wird von innen nach außen verlegt. Auf der körperlichen Ebene gesehen handelt es sich um Vorgänge der Einverleibung und der Ausscheidung; die korrespondierenden Triebkomponenten (Partialtriebe) sind dem oralen bzw. dem analen Körperbereich zugeordnet.

14 Freud S (1923) Das Ich und das Es. SA III, S. 273-330

15 Freud S (1923) Das Ich und das Es. SA III, S. 303 


\subsection{Das Denken, Primär- und Sekundärvorgang}

Bereits 1895, im „Entwurf einer Psychologie“ und im 7. Kapitel der Traumdeutung (1900) hatte Freud sich mit den verschiedenen Funktionsmodi des Denkens auseinandergesetzt. 1911, im zeitlichen Kontext seiner Beschäftigung mit der Wahnbildung im Zuge der Schreber'schen Wahn-Analyse, nahm Freud die Thematik der Differenzierung der Denkvorgänge wieder auf: In den „Formulierungen über die zwei Prinzipien des psychischen Geschehens " ${ }^{16}$ beschreibt er die durch das Ausbleiben von Triebbefriedigung in Gang gesetzte Ergänzung des Lustprinzips durch das Realitätsprinzip als Grundlage für die Ausbildung von Ich-Funktionen wie Aufmerksamkeit, Realitätsprüfung, Urteilsfällung, Merken, Gedächtnis, Denken als Probehandeln, gezieltem Handeln statt ungerichteter motorischer Abfuhr usw.

Der durch die Interaktionsbeziehung zur Außenwelt/Realität entstandene sekundäre Denkmodus des Ichs unterscheidet sich (durch bestimmte diese Realität einbeziehende, einschränkende Prinzipien) von der vom Lustprinzip gesteuerten primären Funktionsweise des Es, dem „Primärvorgang“, der im Traum und bei der Wahnbildung wirksam wird. Dieser sekundäre Denkmodus (Sekundärvorgang) beruht auf stabilen Bedeutungsbesetzungen, die durch die Verknüpfung von Sachvorstellungen mit Wortvorstellungen erreicht werden. Ziel dieser Entwicklung des sekundärprozesshaften Denkens ist schlussendlich - indem der Triebaufschub in Kauf genommen wird - die reale Sicherung der Lustbefriedigung, wobei der Bereich der Phantasie und das Träumen vom Primärvorgang beherrscht werden.

„[...] die bewusste Vorstellung umfasst die Sachvorstellung plus der zugehörigen Wortvorstellung, die unbewusste ist die Sachvorstellung allein. Das System Ubw [Unbewusst] enthält die Sachbesetzungen der Objekte, die ersten und eigentlichen Objektbesetzungen; das System Vbw [Vorbewusst] entsteht, indem diese Sachvorstellung durch die Verknüpfungen mit den ihr entsprechenden Wortvorstellungen überbesetzt wird. " 17

Der Vorgang des Bewusstwerdens einer Vorstellung erfolgt also über die Besetzung mit einer Wortvorstellung, welche als solche „vorbewusst“ und über Aufmerksamkeitsbesetzung schließlich bewusst wird.

„Das Ich ist vom Es nicht scharf getrennt, es fließt nach unten hin mit ihm zusammen“"18, schreibt Freud. Der Austausch zwischen diesen Systemen ermöglicht (abhängig von der Funktionsbereitschaft der Abwehr und anderer Ich-Funktionen) einerseits Phantasie und schöpferische Kreativität. Er ist andererseits Ausgangspunkt für neurotische und psychotische Symptombildung. In der Neurose wird der Realität, mittels Material aus der Phantasiewelt, eine besondere Bedeutung und ein geheimer - symbolischer - Sinn gegeben. In der Psychose, wenn das Übergreifen von Funktionsmechanismen des Es auf das Ich nicht kontrolliert werden kann, setzt sich die Phantasie an die Stelle der Realität; die Realität wird umgebaut. ${ }^{19}$

Einen in diesem Zusammenhang wesentlichen Parameter stellt die Fähigkeit zur Realitätsprüfung dar, eine Funktion des urteilenden und unterscheidenden Denkens.

16 Freud S (1911) Formulierungen über die zwei Prinzipien des psychischen Geschehens, SA III, S. 17-24

17 Freud S (1915) Das Unbewusste. SA III, S. 160

18 Freud S (1923) Das Ich und das Es. SA III, S. 292

19 Freud S (1924) Der Realitätsverlust bei Neurose und Psychose. SA III, S. 355-361 
Im Zusammenhang mit der Verneinung ${ }^{20}$ als dafür erforderliche intellektuelle Voraussetzung zeichnet Freud die Entwicklung der Urteilsfunktion aus ihrem triebhaften Ursprung zur Ichfunktion nach. Er beschreibt dabei die Unterscheidung von Eigenschaften des Objekts („Das will ich essen oder will es ausspucken“" ${ }^{21}$ ) und das Wiederfinden des vorgestellten Objektes in der Wahrnehmung als die Crundlage des Urteilens und der Realitätsprüfung. Die Realitätsprüfung ist also zu verstehen als Überprüfung, ob eine innere Vorstellung, (zunächst die der Mutterbrust als halluzinierte Wunscherfüllung), die einer früher gemachten Wahrnehmung entstammt, in der aktuellen äußeren Realität wiederzufinden ist.

„Das Urteilen ist die zweckmäßige Fortentwicklung der ursprünglich nach dem Lustprinzip erfolgten Einbeziehung ins Ich oder Ausstoßung aus dem Ich. Seine Polarität scheint der Gegensätzlichkeit der beiden von uns angenommenen Triebgruppen zu entsprechen. Die Bejahung - als Ersatz der Vereinigung - gehört dem Eros an, die Verneinung - Nachfolge der Ausstoßung - dem Destruktionstrieb“22, schreibt Freud. „Mittels des Verneinungssymbols macht sich das Denken von den Einschränkungen der Verdrängung frei und bereichert sich um Inhalte, deren es für seine Leistungen nicht entbehren kann." ${ }^{23}$

\subsection{Narzissmus}

Die Besetzung des Ichs mit Libido - als die liebende Zuwendung zu sich selbst - nennt Freud Narzissmus. ${ }^{24}$ Diese Besetzung ist die Voraussetzung für die Fähigkeit, sich selbst zu lieben und aus seinem Ich wie aus einem Reservoir Liebesbesetzungen an andere auszuschicken und wieder zurückzunehmen (wie „ein Protoplasmatierchen seine Pseudopodien“"25). Gleichzeitig wird mit Narzissmus auch jenes frühe Entwicklungsstadium bzw. jene psychische Funktionsebene bezeichnet, in welcher sich über die libidinöse Besetzung die Unterscheidbarkeit von Innenwelt - dem Ich-Selbst - und der Außenwelt - den Objekten - herausbildet. Freud spricht von primärem ${ }^{26}$ Narzissmus und meint damit ein Entwicklungsniveau, in dem das Kind zunächst sich selbst mit seiner ganzen Libido besetzt hat. Der „sekundäre“ Narzissmus wäre dann die Rücknahme der von einer Objektbesetzung abgezogenen Libido auf sich selbst.

Der „Objektlosigkeit“ des primären Narzissmus liegt die noch nicht erfolgte Trennung und Differenzierung von Innen- und Außenwelt zugrunde; so ist sein Denken auch gekennzeichnet von der Allmacht der Gedanken. Freud siedelt dieses Stadium, in welchem sich das Ich als libidinöses Objekt konstituiert, zwischen der von ihm als „objektlos“ postulierten Phase des „Autoerotismus“ und der Fähigkeit zur Objektliebe an.

Eine Fixierung auf narzisstischem Niveau bedeutet eine (partielle) Behinderung in der Verknüpfung der beiden Denkmodi, welche die Einbeziehung des Objekts, des „anderen“, des „realen Außen“ zur Voraussetzung hat. Sie ist Ausdruck der Erfahrung eines Nicht-verfügen-Könnens über eine Bezugsperson („Primärobjekt“ - in der Regel die Mutter, die Eltern), die imstande war, das Unangenehme des Triebaufschubs

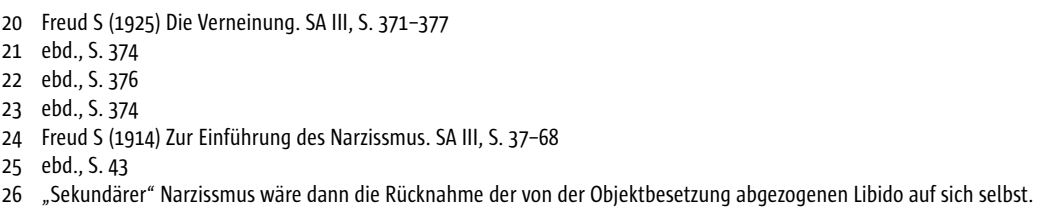


ausreichend erträglich zu machen und damit die Differenzierung von Triebregulation und Strukturbildung zu unterstützen.

„Das Ich ist vor allem ein körperliches, es ist nicht nur ein Oberflächenwesen, sondern selbst die Projektion einer Oberfläche“, sagt Freud an anderer Stelle ${ }^{27}$ und betont die Rolle der äußeren und inneren Körperwahrnehmung für die Ichbildung. ${ }^{28}$ Zwangsläufig müssen diese Wahrnehmungen des Körpers und die Befriedigung seiner Bedürfnisse maßgeblich für die Gestaltung der Libidobesetzung des Ichs bzw. Selbst (primärer Narzissmus) und der bemutternden Person (Primärobjekt) sein, die in der frühen Kindheit die Außenwelt vertritt.

\subsection{Traum und Wahn}

Die Wirkungsweise des Primärvorgangs, welche wir auch bei der Wahnbildung beobachten können, hat Freud am Phänomen des Traumes beschrieben. Der Schlafzustand bietet die dazu erforderlichen Voraussetzungen, nämlich eine weitgehende Herabsetzung der Aufmerksamkeitsbesetzung der Außenwelt, und damit eine Annäherung an einen narzisstischen Zustand als einen weitgehenden „Rückzug der Libidobesetzungen ins Ich“. Damit ist die Ichfunktion der Realitätsprüfung ausgeschaltet bzw. reduziert. Die Nichtbesetzung der Motorik (als Abfuhrorgan gegenüber dem Außen) erlaubt die Lockerung der zensorischen Funktionen der Abwehr. Unter diesen Bedingungen gelangen unerledigte Tageswünsche, „Versagungen“, verstärkt durch assoziierte Triebwünsche aus dem Es, zu einer wie in „Bilderschrift“ (der Schreibweise des Primärvorgangs) dargestellten „halluzinatorischen Wunscherfüllung“. Der manifeste Traum ist das zu Bewusstsein gelangende Erlebnis dieses Vorgangs.

Dabei werden mithilfe der primärprozesshaften Funktionsmechanismen von Verschiebung und Verdichtung, der Darstellung aufgrund von Ähnlichkeiten sowie der Verwendung symbolischer Elemente unbewusste Wunschregungen in einen wunscherfüllenden Ablauf gebracht und mittels der synthetischen Funktion des Ichs zu einem kohärenten und bewusstseinsfähigen manifesten Zusammenhang umgeformt. Durch „darübergelegte“ zensorische Einflüsse des Ichs, die auch im Schlaf - allerdings in verringertem Ausmaß - wirksam sind, ist der latente Trauminhalt mehr oder weniger unkenntlich geworden. Die archaischen Gesetzmäßigkeiten der Stufe, auf der diese Wünsche organisiert sind, bleiben erhalten: Im Primärvorgang gibt es keine Kausalbeziehungen, keine Zeit, keine Unvereinbarkeit von Gegensätzen, keine Verneinung.

Mittels Verknüpfung des manifesten Traumes mit den assoziativen Einfällen des Träumers und Übersetzen der Symbolbedeutungen ist es mehr oder weniger möglich, den latenten Inhalt, d.h. die Versagung und den dahinterstehenden Triebwunsch bzw. die damit zusammenhängende, in die frühe Kindheit zurückreichende Erfahrungs- und Vorstellungswelt zu erkennen, welche sich hier darstellt.

27 ebd., S. 294

28 Hier wird auf Paul Federn verwiesen, der den Begriff „Ichgrenzen“ einführt und die Besetzungsenergie des Ichs als „Ich-Gefühl“ benennt: die Einheit des Erlebens des Individuums in Raum, Zeit, Kausalität, qualitativen und quantitativen Veränderungsmöglichkeiten, wobei in der Entwicklung die einzelnen Körperwahrnehmungen als „Körper-Ich“ in das seelisches Ich integriert werden und beide Bereiche zu einem vollständigen Gesamt-Ichgefühl konsolidiert werden. Er betont die Beschädigung der Ichgrenzen und der Ichfestigkeit und deren Verlust in der Psychose und erklärt Depersonalisation und Entfremdung mit einem Mangel an Libidobesetzung dieser Ichgrenzen (Federn 1978). 
Freud betont immer wieder die Ähnlichkeit dieser Gestaltungselemente bei Wahn-, Phantasie- und Traumbildung. Je weiter die Gestaltungselemente in die frühe Vorstellungswelt der Kindheit zurückreichen, umso deutlicher werden ihre Bezüge zum Körperlichen, zu Körperteilen und den damit korrespondierenden Partialtrieben bzw. Partialobjekten und den damit verknüpften Phantasien.

„Das Träumen ist ein Stück des überwundenen Kinderseelenlebens. In den Psychosen werden diese sonst im Wachen unterdrückten Arbeitsweisen des psychischen Apparats sich wiederum Geltungerzwingen und dann ihre Unfähigkeit zur Befriedigung unserer Bedürfnisse gegen die Außenwelt an den Tag legen [...]", schreibt Freud in der „Traumdeutung“. ${ }^{29}$ Und weiter: „Die unbewussten Wunschregungen streben offenbar auch bei Tag, sich geltend zu machen, und die Tatsache der Übertragung sowie der Psychosen belehren uns, dass sie aufdem Wege durch das System des Vorbewussten zum Bewusstsein und zur Beherrschung der Motilität vordringen möchten. In der Zensur zwischen Ubw [Unbewußtem] und Vbw [Vorbewußtem], deren Annahme uns der Traum geradezu aufnötigt, haben wir also den Wächter unserer geistigen Gesundheit zu erkennen und zu ehren. [...] Minder harmlos gestaltet es sich, wenn die Kräfteverschiebung nicht durch den nächtlichen Nachlass im Kräfteaufwand der kritischen Zensur, sondern durch pathologische Schwächung derselben oder durch pathologische Verstärkung der unbewussten Erregungen hergestellt wird, solange das Vorbewusste besetzt und die Tore zur Motilität offen sind. Dann wird der Wächter überwältigt, die unbewussten Regungen unterwerfen sich das Vbw, beherrschen von ihm aus unser Reden und Handeln und lenken den nicht für sie bestimmten Apparat vermöge der Anziehung, welche die Wahrnehmungen auf die Verteilung unserer psychischen Energie ausüben. Diesen Zustand heißen wir Psychose.“" ${ }^{30}$

Freud beschreibt das Grundelement einer psychischen Leistung analog des Reflexbogens, der von einem Wahrnehmungsreiz (vom „sensorischen Ende“) ausgeht und in ein „motorisches Ende“ mündet. Differenzierungen am sensorischen Pol werden zu Erinnerungsspuren, in denen sich gleichzeitige Wahrnehmungen verknüpfen. Die solcherart entstehenden Erinnerungssysteme sind die Grundlage der Assoziation.

Der Ablauf eines progredienten psychischen Vorgangs erfolgt von diesen durch aktuelle Afferenzen aktivierten Erinnerungssystemen des unbewussten Es-Bereichs aus über die Verknüpfung von Sachvorstellungen mit Wortvorstellungen (sekundärer Denkmodus) hin zum vorbewussten Ich-Bereich, womit bei entsprechender Aufmerksamkeitsbesetzung die Umsetzung in bewusstes Denken (= „Probehandeln“) bzw. willkürliche Motilität ermöglicht ist.

Das absichtliche Erinnern wäre anhand dieses Modells als regrediente Bewegung vom motorischen Pol zum sensorisches Pol innerhalb der Systeme „Bewusst“ und „Vorbewusst" zu verstehen.

Die halluzinatorische Belebung von Wahrnehmungsbildern bzw. die Beweglichkeit der assoziativen Verknüpfungen und leichte Ersetzbarkeit der Objekte, wie sie bei der Traumbildung und in psychotischen Zuständen auftreten, bedürfen aber zusätzlicher Bedingungen: Es müssen die sekundärprozesshaften Denkmodi durch primärprozesshafte ersetzt werden, damit die Gedanken in ihr „Rohmaterial“ aufgelöst werden können. Dies entspricht im oben angeführten Reflexbogenmodell einer Verschiebung des gesamten Geschehens in Richtung sensorisches Ende. Freud sieht als Vorausset-

29 Freud S (1900) Die Traumdeutung. SA II, S. 540

30 ebd., S. 540 f. 
zung dieser Regression einen (partiellen) Abzug der Libido von der Außenwelt an. Er beobachtet jedoch, dass dieser Vorgang nie ein absoluter zu sein scheint, auch nicht bei den Psychosen:

„Das Problem der Psychose wäre einfach und durchsichtig, wenn die Ablösung des Ichs von der Realität restlos durchführbar wäre. Aber das scheint nur selten, vielleicht niemals vorzukommen. Selbst von Zuständen, die sich von der Außenwelt so weit entfernt haben wie die einer halluzinatorischen Verworrenheit (Amentia), erfährt man durch die Mitteilung der Kranken nach ihrer Genesung, dass damals in einem Winkel ihrer Seele, wie sie sich ausdrücken, eine normale Person sich verborgen hielt, die den Krankheitsspuk wie ein unbeteiligter Beobachter an sich vorüberziehen ließ." ${ }^{31}$

Freud beschreibt auch die Beobachtung, dass ein wahnhafter Inhalt im Traum vorhanden sein kann, während im Wachbewusstsein davon (noch) nichts feststellbar ist und dass umgekehrt im wahnhaften Zustand der Traum die eigentliche Realität abbilden kann. Er erklärt dies damit, dass sich „der Traum vom Wachdenken dadurch [unterscheidet], dass er Inhalte [aus dem Bereich des Verdrängten] aufnehmen kann, die im Wachdenken nicht vorkommen dürfen“. ${ }^{32}$

In diesem Zusammenhang beschreibt Freud einen Patienten, der seine klassischen Verfolgungsgedanken quasi unter rationalem Verschluss halten konnte, sodass sie in der Analyse nur gelegentlich „aufblitzten“. Sichtbar war ein generelles Misstrauen in realen Beziehungen zu Männern. Er bemerkt dazu: „Das mag in vielen Fällen von Paranoia ähnlich vorkommen, und wenn so eine Erkrankung losbricht, halten wir vielleicht die geäußerten Wahnideen für Neuproduktionen, während sie längst bestanden haben." 33

\subsection{Der „Fall Schreber“34}

Freud hatte die Paranoia bereits in den Briefen an Fließ 1895/96 und 1896 in seiner Abhandlung „Weitere Bemerkungen über Abwehrneuropsychosen“" 35 als eine durch einen Abwehrvorgang bedingte Erkrankung, d.h. als „Abwehrneuropsychose“ “ ${ }^{66}$ definiert und die Projektion als ihren Hauptmechanismus beschrieben. $1911^{37}$ nimmt er die autobiografischen Aufzeichnungen Daniel Paul Schrebers über dessen jahrelanges, systematisiertes Wahnsystem zur Grundlage einer Analyse und entwickelt daraus Aussagen über die dynamischen Abläufe bei Wahnbildung und Psychosen.

Daniel Paul Schreber, den ehemaligen sächsischen Senatspräsidenten, würden heute aufgrund der Gesamtsymptomatik als an einer schizophrenen Psychose erkrankt diagnostiziert werden. Schreber beschreibt in einem 1903 publizierten Buch ${ }^{38}$ seine sich über Jahre hinziehenden mannigfaltigen (Wahn-)Vorstellungen. Der Beginn des Geschehens ist eine durch berufliche Überforderung ausgelöste Krise, in der er auf die Hilfe eines ihm bereits von einer früheren Gelegenheit bekannten Arztes, Dr. Flech-

31 Freud S (1949a) Abriss der Psychoanalyse. GW I, S. 132

32 Freud S (1922) Neurotische Mechanismen bei Eifersucht, Paranoia und Homosexualität. SA VII, S. 225

33 ebd., S. 224

34 Niederland WG (1978)

35 Freud S (1896b) Weitere Bemerkungen über die Abwehr-Neuropsychosen. GW I, S. 377

36 Zur Bedeutung des Begriffs „Abwehrneuropsychose“ siehe May-Tolzmann 1996

37 Freud S (1911) Psychoanalytische Bemerkungen über einen autobiographisch beschriebenen Fall von Paranoia. SA VII, S. 133-204

38 Schreber DP (1903) 
sig, hofft. Nachdem er sich zunächst - in der akuten psychotischen Phase - homosexuellen Angriffen dieses Arztes ausgesetzt wähnt, tritt in einer fortschreitenden Entwicklung und Systematisierung des Wahngebäudes Gott an die Stelle des Arztes. Die Vorstellungen, Gott für wollüstige Zwecke zur Verfügung zu stehen und durch die(Er-) Zeugung einer neuen Menschenrasse die Erlösung der Welt zu ermöglichen, machen die damit verbundene Kastrationsphantasie (Verwandlung in eine Frau) nun zu einer logischen und durch die narzisstische Bedeutungsgebung annehmbaren Konsequenz.

Schreber möchte so potent sein, wie er meint, dass sein Vater gewesen sei. Auf der narzisstischen Ebene bedeutet Potenz „Omnipotenz“, Gott-gleichheit, Unverletzbarkeit, Überlegenheit, Unbesiegbarkeit, Allwissenheit etc. Die unbewusste homosexuelle Phantasie ist, durch sexuelle Vereinigung mit dem Vater diese Omnipotenz, den übermächtigen Phallus des Vaters, von diesem bekommen und für sich zur Verfügung zu haben. Als Bedingung für die Erfüllung des Wunsches, vom Vater geliebt zu werden, wird in der Wahnvorstellung die Verwandlung in eine Frau, also die Kastration, dargestellt.

Freud erkennt als Ausgangspunkt der Symptombildung des Wahns (wie bei den Neurosen) eine Versagung, d.h. einen nicht erfüllten Triebwunsch. Diese aktuelle Versagung, welche als Wiederholung früherer Versagungen erlebt wird, hat eine Regression der Triebdynamik auf einen frühen Fixierungspunkt der kindlichen Entwicklung zur Folge. Fixierung bedeutet, dass in der Entwicklung von Triebkomponenten eine Hemmung stattgefunden hat, die zu einem teilweisen Verbleiben der Libidoorganisation auf dieser Entwicklungsstufe führt. „Unsere Dispositionen sind also Entwicklungshemmungen, "39 schreibt Freud.

Zum Zeitpunkt der aktuellen Versagung resultiert daher die Notwendigkeit, die Abwehr von psychischen Abkömmlingen jener primär zurückgebliebenen Triebe und damit verknüpften Strebungen zu bewältigen. Bei Misslingen dieser Abwehr erfolgt als manifester Ausdruck jener Strebungen der Durchbruch, die „Wiederkehr des Verdrängten" von der Stelle der Fixierung her.

Als disponierenden Fixierungspunkt für die Paranoia ${ }^{40}$ ortet Freud den Narzissmus als die „zwischen Autoerotismus und Objektwahl vermittelnde Entwicklungsphase“: Die Regression auf einen Fixierungspunkt „,vor der Objektwahl“ bedeutet den Rückzug der Libido von der Außenwelt, von vorher geliebten Personen in das Ich. Auf dieser Stufe der Regression sind Ich und Welt noch nicht voneinander zu unterscheiden. (Freud sieht diesen Aspekt als Erklärung des Phänomens des Größenwahns an.) Der Wahn als die sichtbare Symptombildung wird indessen als Heilungsversuch, als IchLeistung verstanden. Dabei dient die Wahnbildung dazu, mit der Wiederkehr der verdrängten Triebwünsche zurechtzukommen und sie in einem Gleichgewicht von Befriedigung und Abwehr zu halten. Dies erfolgt auf projektivem Wege, das „innerlich Aufgehobene kehrt von außen wieder“. ${ }^{41}$ Die äußere Realität wird ersetzt durch innere Vorstellungen, die als real erlebt werden, wobei sie der aktuellen Realitätsprüfung enthoben sind.

39 Freud S (1913) Disposition zur Zwangsneurose. SA VII, S. 110

40 ebd. (über die Schizophrenie - von Freud als „Paraphrenie“ bezeichnet - und die Paranoia): „Die ihnen beiden eigentümlichen Charaktere des Größenwahns, der Abwendung von der Welt der Objekte und der Erschwerung der Übertragung haben uns zum Schlusse genötigt, dass deren disponierende Fixierung in einem Stadium der Libidoentwicklung vor der Herstellung der Objektwahl, also in der Phase des Autoerotismus und des Narzissmus zu suchen ist."

41 Freud S (1911) Psychoanalytische Bemerkungen über einen autobiographisch beschriebenen Fall von Paranoia. SA VII, S. 194 
Die verschiedenen Wahnformen sind im Zusammenhang mit der narzisstischen Ebene als Abwehr einer in der Projektion verdeckt dargestellten unbewussten homosexuellen Wunschphantasie zu verstehen, deren Verdrängung bzw. bisherige Sublimierung in soziale Strebungen durch die Regression aufgehoben worden ist. Die Abwehr dieses homosexuellen Triebwunsches bei der Wiederkehr des Verdrängten erfolgt durch Projektion und Widerspruch und gestaltet die Symptombildung. So ist Schreber überzeugt, dass

„Gott ein den weltordnungsgemäßen Daseinsbedingungen der Seelen beständiges Genießen [verlangt]; es ist meine Aufgabe, ihm dasselbe [...] in der Form ausgiebigster Entwicklung der Seelenwollust zu verschaffen; soweit dabei für mich etwas vom sinnlichen Cenusse abfällt, bin ich berechtigt, denselben als eine kleine Entschädigung für das Übermaßder Leiden und Entbehrungen, das mir seit Jahren auferlegt ist, mitzunehmen [...]“.42

Die folgenden möglichen Variationen der Abwehr des Satzes „Ich liebe ihn“ bilden die Hauptformen der Paranoia (s. Tab. 3). ${ }^{43}$

Tab. 3 Variationen der Abwehr des Satzes „Ich liebe ihn“

\begin{tabular}{llll} 
Ich & liebe & ihn & Wahn \\
Er (nicht ich) & hasst/verfolgt & mich & Verfolgungswahn \\
\hline Ich & liebe & sie (nicht ihn), weil sie mich liebt & Erotomanie \\
\hline Sie (nicht ich) & liebt & ihn & Eifersuchtswahn \\
\hline Ich & liebe nicht & und niemanden (nur mich) & Größenwahn \\
\hline
\end{tabular}

Den Grund für die Affektumkehr ortet Freud im Vorhandensein gegensätzlicher Gefühlsanteile in einer Objektbeziehung, in welcher die ambivalente Beziehung zum gleichgeschlechtlichen Elternteil wiederbelebt wird. Er schreibt, dass

„die Feindseligkeit, die der Verfolgte bei den anderen findet, der Widerschein der eigenen feindseligen Gefühle gegen diese anderen ist. Da wir wissen, dass beim Paranoiker gerade die geliebteste Person des gleichen Geschlechtes zum Verfolger wird, entsteht die Frage, woher diese Affektumkehrung rührt, und die nahe liegende Antwort wäre, dass die stets vorhandene Gefühlsambivalenz die Grundlage für den Hass abgibt und die Nichterfüllung der Liebesansprüche an ihn verstärkt“. ${ }^{44}$

Offenbar wird durch die Liebesversagung die bislang durch Reaktionsbildung abgewehrte feindselige Seite der Ambivalenz reaktiviert. (Soziale Gefühle entstehen über Identifizierung, nachdem die aggressiv-feindselige, rivalisierende Einstellung durch eine Reaktionsbildung ${ }^{45}$ gegen die Aggressionsimpulse abgelöst worden ist. ${ }^{46}$ )

Es ist das narzisstische Niveau der Fixierung, die Abkehr von der Realität als Ausdruck eines weitreichenden Libidoabzugs von der Außenwelt, welche - aus der Sicht der Libidodynamik - den Mechanismus der Neurose von jenem der Psychose unterscheidet.

42 ebd., S. $160 f$.

43 ebd., S. $186 \mathrm{ff}$.

44 Freud S (1922) Neurotische Mechanismen bei Eifersucht, Paranoia und Homosexualität. SA VII, S. 222

45 Bei der Reaktionsbildung wird ein (unbewusster) Triebimpuls durch eine ihm entgegengesetzte Verhaltensweise abgewehrt.

46 Freud S (1921) Massenpsychologie und Ich-Analyse. SA IX, S. 113; Freud S (1922) Über einige neurotische Mechanismen bei Eifersucht, Paranoia und Homosexualität. SA VII, S. 227 
„In dem Maße, als das umstrittene Objekt das wichtigste in der Außenwelt wird, einerseits alle Libido an sich ziehen will, andererseits alle Widerstände gegen sich mobil macht, wird der Kampf umseinzelne Objekt mit einer allgemeinen Schlacht vergleichbar, in deren Verlauf sich der Sieg der Verdrängung durch die Überzeugung ausdrückt, die Welt sei untergegangen und das Selbst sei allein übergeblieben.“ " ${ }^{7}$ „Der Kranke [Schreber] hat den Personen seiner Umgebung und der Außenwelt überhaupt die Libidobesetzung entzogen, die ihnen bisher zugewendet war; damit ist für ihn alles gleichgültig und beziehungslos geworden und muss durch sekundäre Rationalisierung als, hingewundert, flüchtig hingemacht erklärt werden. Der Weltuntergang ist die Projektion dieser inneren Katastrophe; seine subjektive Welt ist untergegangen, seitdem er ihr seine Liebe entzogen hat: [...] Und der Paranoiker baut sie wieder auf, nicht prächtiger zwar, aber so, dass er wieder in ihr leben kann. Er baut sie auf durch die Arbeit seines Wahns. Was wir für die Krankheitsproduktion halten, die Wahnbildung, ist in Wirklichkeit der Heilungsversuch, die Rekonstruktion. Diese gelingt nach der Katastrophe mehr oder weniger gut, niemals völlig [...] Der eigentliche Verdrängungsvorgang besteht in einer Ablösung der Libido von vorher geliebten Personen - und Dingen. Er vollzieht sich stumm [...] Was sich lärmend bemerkbar macht, das ist der Heilungsvorgang, der die Verdrängung rückgängig macht und die Libido wieder zu den von ihr verlassenen Personen zurückführt. Er vollzieht sich bei der Paranoia auf dem Weg der Projektion. [...] das innerlich Aufgehobene [kehrt] von außen wieder." ${ }^{48}$

Freud plädiert dafür,

„die Paranoia als selbständigen klinischen Typus aufrechtzuerhalten, auch wenn ihr Bild noch so häufig durch schizophrene Züge kompliziert wird“.49 „Der Ausgang der Dementia präcox (Schizophrenie) [...] ist im allgemeinen ungünstiger als der der Paranoia; [...] Die Regression geht nicht nur bis zum Narzissmus, sondern bis zur vollen Auslassung der Objektliebe und Rückkehr zum infantilen Autoerotismus. Die disponierende Fixierung muss also weiter zurückliegen als die der Paranoia, im Beginn der Entwicklung, dievom Autoerotismus zur Objektliebestrebt, enthalten sein [...] Es könnenja in der Entwicklung mehrere Fixierungen zurückgelassen worden sein und der Reihe nach den Durchbruch der abgedrängten Libido gestatten, etwa die später erworbene zuerst und im weiteren Verlauf der Krankheit dann die ursprüngliche, dem Ausgangspunkt näher liegende. "so

\subsection{Neurose und Psychose, die Verleugnung der Realität}

In Weiterentwicklung der Überlegungen zur Symptombildung präzisiert Freud den Unterschied zwischen Neurose und Psychose. Wie bei der Neurose gebe es zwei erforderliche Schritte für die Bildung der Krankheitszeichen:

1. die Abwehr eines unerträglichen Versagungserlebens in der frühen Kindheit und

2. die Wiederkehr des Verdrängten im Zusammenhang mit dem Misslingen der Abwehr im Rahmen einer aktuellen Versagung.

47 Freud S (1911) Psychoanalytische Bemerkungen über einen autobiographisch beschriebenen Fall von Paranoia. SA VII, S. 195

48 ebd., S. $192 f$.

49 ebd., S. 197

50 ebd., S. $198 f$. 
Bei der Psychose werde im ersten Schritt (als frühkindliche Disponierung) nicht wie bei der Neurose (nur) die Triebregung verdrängt, sondern die Realität (bzw. Teile der Realität) geleugnet. Zugunsten des Es, des Triebwunsches, wird also die Realitätsprüfung aufgegeben:

„Bei der Psychose ruht der Akzent ganz auf dem ersten Schritt (der Leugnung der Realität, Anm. d. Verf.), der an sich krankhaft ist und nur zum Kranksein führen kann, bei der Neurose hingegen auf dem zweiten, dem Misslingen der Verdrängung, während der erste Schritt (die Verdrängung) gelingen kann und auch im Rahmen der Gesundheit unzählige Male gelungen ist, wenn auch nicht ganz ohne Kosten zu machen [...]“51

Der zweite Schritt (im Zusammenhang mit dem Triebdruck der aktuellen Versagung) erfolgt nun bei der Psychose nicht durch eine

„Einschränkung des Es wie bei der Neurose auf Kosten der Realbeziehung (durch Vermeidung der Realität, Anm. der Verf.), sondern auf einem anderen, mehr selbstherrlichen Weg durch Schöpfung einer neuen Realität, welch nicht mehr den nämlichen Anstoßbietet wie die Verlassene. [...] Es ist kaum zweifelhaft, dass die Phantasiewelt bei der Psychose die nämliche Rolle spielt [wie bei der Neurose; E. H.], dass sie auch hier die Vorratskammer darstellt, aus der derStoffoder die Muster für den Ausbau der neuen Realität geholt werden. Aber die neue, phantastische Außenwelt der Psychose will sich an die Stelle der äußeren Realität setzen, die der Neurose hingegen lehnt sich wie ein Kinderspiel gern an ein Stück der Realität an [...]“.52

\subsection{Das konkretistische Denken}

Der Schizophrene setzt abstrakte Dinge mit konkreten Dingen gleich. Dieser Konkretismus bildet den besonderen Umgang mit der Realität ab. Freud erklärt das Phänomen damit, dass hier der Weg zum Objekt über den Wortanteil desselben erfolgt. ${ }^{53} \mathrm{Im}$ psychotischen Denkmodus werden Wortvorstellungen wie Sachvorstellungen behandelt und die Funktionsweisen des realitätsgerechteren Sekundärvorgangs durch primärprozesshafte Mechanismen ersetzt (anstatt in flexibler Weise miteinander zu kooperieren und einander produktiv zu beeinflussen). In anderen Worten ausgedrückt heißt das, dass bei dieser Form des Denkens statt eines (symbolischen) Vergleichs („,so wie“) eine Gleichsetzung hergestellt wird, womit Korrektur- und Differenzierungsmöglichkeiten reduziert bzw. ausgeschaltet sind und die objektive Realität durch eine (Phantasie-)Vorstellung ersetzt wird. Dies trägt zur Unkorrigierbarkeit des Wahns bei.

\subsection{Der Wahn als Folge einer Spaltung im Ich}

„Der Wahn (wird) wie ein aufgesetzter Fleck dort gefunden, wo ursprünglich ein Einriss in der Beziehung des Ichs zur Außenwelt entstanden war“, formuliert Freud $1924^{54}$ und definiert damit den Wahn als Folge einer dauerhaften Beschädigung der Funktionseinheit Ich. Den dafür verantwortlichen Mechanismus bezeichnet er später als „Ich-Spaltung im Abwehrvorgang“ (1940). Anders als bei der Verdrängung, welche

51 Freud S (1924e) Der Realitätsverlust bei Neurose und Psychose. SA III, S. 360

52 ebd., S. 358, 361

53 Freud S (1915) Das Unbewusste. SA III, S. 162

54 Freud S (1924b) Neurose und Psychose. SA III, S. 335 
dazu führt, dass eine konflikthafte (Trieb-)Vorstellung unbewusst gehalten wird, wird hier der Konflikt zwischen einem Triebanspruch und seiner Versagung durch die Außenwelt dadurch gehandhabt, dass die Realität gleichzeitig anerkannt und verleugnet wird: „Beide streitenden Parteien haben ihr Teil bekommen; der Trieb darf seine Befriedigung behalten, der Realität ist der gebührende Respekt gezollt worden. Aber umsonst ist bekanntlich nur der Tod. Der Erfolg wurde erreicht auf Kosten eines Einrisses im Ich, der nie wieder verheilen, aber sich mit der Zeit vergrößern wird. “55

Diese Ich-Spaltung als Folge der Verleugnung entdeckt Freud zunächst als Erklärung des Phänomens des Fetischismus. ${ }^{56}$ Der Fetisch dient als bewusst verwendetes Element mit unbewusstem Bedeutungs- und Wunscherfüllungscharakter, um die „Kastration“, also die Penislosigkeit des weiblichen Geschlechts, zu leugnen. Freud betont, dass das Phänomen dieses Vorgangs nicht auf den Fetischismus zu beschränken sei. Im seiner letzten, posthum veröffentlichten Schrift „Abriss der Psychoanalyse“ 57 nimmt er das Thema der Ich-Spaltung im Zusammenhang mit der psychotischen Disposition wieder auf:

Neben der,Erledigung unliebsamer [innerer] Triebansprüche durch Verdrängung“ komme das kindliche Ich „oft genug in die Lage, sich einer peinlich empfundenen Zumutung der Außenwelt zu erwehren, was durch die Verleugnung der Wahrnehmungen geschieht [...] Solche Verleugnungen fallen sehr häufig vor, nicht nur beim Fetischisten, und wo immer wir in die Lage kommen, siezu studieren, erweisen sie sich als halbe Maßregeln, unvollkommene Versuche zur Ablösung von der Realität. Die Ablehnung wird jedes Mal durch eine Anerkennung ergänzt, es stellen sich immer zwei gegensätzliche, voneinander unabhängige Einstellungen her, die den Tatbestand einer Ichspaltung ergeben. Der Erfolg hängt wiederum davon ab, welche von beiden die größere Intensität an sich reißen kann [...] Die beiden [Einstellungen] bestehen nebeneinander. Ist oder wird die letztere die stärkere, so ist damit die Bedingung der Psychose gegeben. Kehrt sich das Verhältnis um, so ergibt sich eine anscheinende Heilung der Wahnkrankheit. In Wirklichkeit ist sie nur ins Unbewusste zurückgetreten, wie man ja auch aus zahlreichen Beobachtungen erschließen muss, dass der Wahn lange Zeit fertig gebildet lag, ehe er manifest zum Durchbruch kam. [...] Wenn wir bisher immer wieder betonen mussten, das Ich verdanke seine Entstehung wie die wichtigsten seiner erworbenen Charaktere der Beziehung zur realen Außenwelt, so haben wir uns daraufvorbereitet, anzunehmen, dass die Krankheitszustände des Ichs, in denen es sich dem Es wiederum am meisten annähert, durch Aufhebung oder Lockerung dieser Außenweltsbeziehung begründet sind [...]“. Der Anlass für den Ausbruch einer Psychose sei entweder, ,dass die Realität unerträglich schmerzhaft geworden ist, oder dass die Triebe eine außerordentliche Verstärkung gewonnen haben, was bei den rivalisierenden Ansprüchen von Es und Außenwelt an das Ich die gleiche Wirkung erzielen muss". ${ }^{8}$

\subsection{Der Wahrheitskern des Wahns}

Freud hat seine Skepsis betreffend die Beeinflussungsmöglichkeiten psychotischer Erkrankungen auf psychologischem Wege immer wieder betont und auf zukünftige Möglichkeiten von chemischen Mitteln gehofft. Zuletzt scheint sich diese Einstel-

55 Freud S (1940e) Die Ichspaltung im Abwehrvorgang. SA III, S. 391 .

56 Freud S (1927) Fetischismus. SA III, S. 379-388

57 Freud S (1949a) Abriss der Psychoanalyse. GW XVII, S. 63-138

58 ebd. S. $134,133,132$ 
lung zu relativieren. Er verweist wieder auf die Verwandtschaft des paranoiden Mechanismus mit dem Traum, vermutet einen Zusammenhang der Unkorrigierbarkeit des Wahns mit einer „Wieder-Erinnerung“, wie er sie bereits bei den Neurosen (Reminiszenz) festgestellt hatte, und sieht hier einen Ansatzpunkt für die therapeutische Arbeit:

„Wir betonen im Mechanismus einer Wahnbildung in der Regel nur zwei Momente, die Abwendung von der Realität und deren Motive einerseits und den Einfluss der Wunscherfüllung auf den Inhalt des Wahns andererseits. Aber kann der dynamische Vorgang nicht eher der sein, dass die Abwendung von der Realität vom Auftrieb des Verdrängten ausgenützt wird, um seinen Inhalt dem Bewusstsein aufzudrängen, wobei die bei diesem Vorgang erregten Widerstände und die Tendenz zur Wunscherfüllung sich in die Verantwortlichkeit für die Entstellung und Verschiebung des Wiedererinnerten teilen. Das ist doch auch der uns bekannte Mechanismus des Traumes, den schon uralte Ahnung dem Wahnsinn gleichgesetzt hat. [...] Man würde die vergebliche Mühe aufgeben, den Kranken vom Irrsinn seines Wahns, von seinem Widerspruch zur Realität, zu überzeugen, und vielmehr in der Anerkennung des Wahrheitskerns einen gemeinsamen Boden finden, auf dem sich die therapeutische Arbeit entwickeln kann. Diese Arbeit bestünde darin, das Stück historischer Wahrheit von seinen Entstellungen und Anlehnungen an die reale Gegenwart zu befreien und es zurechtzurücken an die Stelle der Vergangenheit, der es zugehört." ${ }^{59}$

\subsection{Zusammenfassung}

Bei der Wahnbildung erfolgt, ausgelöst durch eine aktuelle Versagung, ein weitreichender Abzug der Libido von der Außenwelt, was einer Regression auf ein narzisstisches Fixierungsniveau entspricht. „[...] seine subjektive Welt ist untergegangen, seitdem er ihr seine Liebe entzogen hat". ${ }^{60}$ Der Wahn ist als ein Restitutionsversuch dieses Beziehungsverlustes zu sehen. Die dem narzisstischen Niveau entsprechenden homosexuellen Strebungen werden in der Wahnbildung projektiv dargestellt und als Folge ambivalenter Gefühlsstrebungen gegen sich gerichtet wahrgenommen.

Die Vorbedingung (Disposition) für diese aktuelle psychische Reaktion sind traumatisch wirksame Abwehrereignisse in der frühen Kindheit. Dabei hat zur Bewältigung unerträglicher Triebspannung statt der Verdrängung des Triebwunsches eine Leugnung der Realität stattgefunden. Die Folge dieser Verleugnung der Realität ist eine Ich-Spaltung, welche die Etablierung einer Gleichzeitigkeit von „es ist“ und „es ist nicht“ ohne Widerspruch zulässt. Daraus resultiert eine Störung in der weiteren Entwicklung dieses Ichs, das ja mit seinen Funktionen das Vermittlungsorgan zur Außenwelt darstellt. Freud spricht von einem „Einriss im Ich“, einer Beschädigung, die an sich eine Pathologie darstellt, weil sie ein ungesteuertes Übergreifen des primären Denkmodus, der Widerspruch nicht kennt, auf das Denken im Wachbewusstsein ermöglicht. Das führt dazu, dass die Wahrnehmung der realen Objektwelt mit von ihr nicht unterscheidbaren Phantasien durchsetzt ist bzw. ersetzt werden kann.

Die aktuelle Versagung, die die Wahnproduktion auslöst, wird wegen ihres unbewussten Zusammenhangs mit der frühen Versagungssituation als unerträglich erlebt. Im

59 Freud S (1937d) Konstruktionen in der Analyse. GW XVI, S. $54 f$.

60 Freud S (1911) Über einen autobiographisch beschriebenen Fall von Paranoia. SA VII, S. 193 
Zustand der Regression des Ichs werden aufgrund der nun zutage tretenden frühen Ich-Spaltung unter Aufgabe des Objektbezugs primärprozesshafte Denkmodi zur Triebbewältigung eingesetzt: Analog der Traumbildung wird die innere Realität an die Stelle der äußeren Realität gesetzt und der wunscherfüllende Inhalt unter Einsatz primärprozesshafter Mechanismen wie Verschiebung, Verdichtung, szenischer Darstellung und sekundärer Rationalisierung entstellt. Die konkretistische Verwendung der Sprache ist Ausdruck der verlorenen Verankerung im realen objektalen Bezug.

Hinsichtlich des „Wahrheitskerns“ des Wahns liegt ein Potenzial in der Möglichkeit, im Wahnnarrativ über die dargestellte wahnhafte Ersatzwelt Elemente der geleugneten, überfordernden, frühkindlichen Realität zu erkennen und die Schmerzhaftigkeit der frühen Verletzung nachträglich zu würdigen und von der aktuellen Versagung abzugrenzen.

\subsection{Fallprotokolle}

Beim Versuch, die beschriebenen Überlegungen anhand der vorliegenden Wahnprotokolle nachzuvollziehen und diese (soweit dies ohne weitere Mithilfe der Protagonisten möglich ist) wie einen Traum zu deuten, können zunächst einige Unterschiede festgestellt werden:

Der Bericht von Patientin 2 (s. Anhang) beschreibt einen zeitlich begrenzten psychotischen Ablauf, eine psychotische Episode. Als aktuell auslösende Versagung wird die Nichterfüllung eines Liebeswunsches klar erkennbar, wobei wir nicht erfahren, ob der Geliebte je von der an ihn gerichteten Liebessehnsucht Kenntnis erlangt hat. Der Bericht endet mit dem Hinweis auf eine Veränderung hin zu einer depressiven psychischen Verfassung mit einem noch länger bestehenden, dann aber ebenfalls (bewusst) aufgegebenen wahnhaften Rest (den „Nobelpreis für die Liebe zur Philosophie“ erhalten zu haben). Diese Vorstellung, in der sich ein humorvoller Aspekt (der Ansatz einer Sublimierung) andeutet, könnte man als poetische Beschreibung des Realitätskerns der Wahnbildung verstehen: als verdichteten Ausdruck der Liebesenergie und der unerfüllten Liebeswünsche der Erzählerin, die in der Philosophie eine Sublimierungsmöglichkeit mit Einfluss auf ihre Berufswahl gefunden hat; als Darstellung ihrer Sehnsucht, sich in ihren Qualitäten erkannt und wahrgenommen zu fühlen, die den stattgehabten Mangel einer solchen verinnerlichten Erfahrung widerspiegelt; und als eigenes Wissen um ihre Liebe zum „Philosophieren“, also zur Abstraktion, welche dem Wort und der Phantasie den Vorzug vor der realen Welt und ihren Frustrationen gibt. ${ }^{61}$ Die Libidoregression im Wahnprotokoll ist als Bewegungsabfolge nachvollziehbar, als Verarbeitung des aktuellen Versagungserlebnisses erkennbar und kann anhand einzelner Elemente im zeitlichen Ablauf der Wahnbildung interpretiert werden.

Im Gegensatz dazu imponiert der Wahnbericht von Patient 1 (s. Anhang) als Überlagerung einer Vielzahl entsprechender Abläufe. Es entsteht dadurch der Eindruck einer viel komplexeren, für den Betrachter schwer nachvollziehbaren, mit Brüchen ver-

61 Freud weist darauf hin, dass wir im abstrakten Denken in Gefahr geraten, „[...] die Beziehungen der Worte zu den unbewussten Sachvorstellungen zu vernachlässigen, und es ist nicht zu leugnen, dass unser Philosophieren dann eine unerwünschte Ähnlichkeit in Ausdruck und Inhalt mit der Arbeitsweise der Schizophrenien gewinnt“ (Das Unbewusste [1915]. SA III, S. 162). 
sehenen Parallelwelt. Sie scheint die Folge einer jahrelangen Krankengeschichte mit rezidivierenden psychotischen Schüben zu sein, wo eine Remission mit Distanzierung zu den Wahninhalten in der Regel immer weniger gelingt.

In beiden Darstellungen wird die narzisstische Wunschebene deutlich: groß, mächtig, anerkannt, berühmt, ein Held oder Nobelpreisträgerin zu sein, in anderen Worten: alle Aufmerksamkeit und Wertschätzung seitens des Liebesobjektes/der Welt für sich zu haben - als Pendant für die Wichtigkeit, die das Liebesobjekt, die „ganze Welt“ repräsentierend, für das Kleinkind bedeutet. Freud sagt, die Wahnproduktion (nicht die anderen Symptome der Psychose, nur der spezielle psychische Vorgang des wahnhaften Denkens) hänge mit einem Fixierungspunkt auf ein bestimmtes Niveau der kindlichen psychischen Entwicklung zusammen, welches durch die narzisstische Form der gleichgeschlechtlichen Objektbeziehung charakterisiert ist. Wir finden diesen homosexuellen Beziehungsaspekt in beiden Wahnberichten in unterschiedlicher Ausprägung. Wie Freud am Ende seiner Schreber-Analyse schreibt,

[...] ist [es] auch keineswegs wahrscheinlich, dass die homosexuellen Anstöße, die wir bei der Paranoia so häufig, vielleicht regelmäßig finden, in der Ätiologie der weit uneingeschränkteren Dementia präcox eine ähnlich bedeutsame Rolle spielen.

Unsere Annahmen über die disponierenden Fixierungen bei Paranoia und Paraphrenie ${ }^{62}$ machen es ohne weiteres verständlich, dass ein Fall mit paranoischen Symptomen beginnen und sich doch zur Demenz ${ }^{63}$ entwickeln kann, dass paranoide und schizophrene Erscheinungen sich in jedem Ausmaßkombinieren". ${ }^{64}$

Sehr eindrucksvoll kommt in beiden Protokollen der konkretistische Denkmodus zur Darstellung, wobei Wortvorstellungen wie Realitäten behandelt werden und auf diese Weise die aktuelle Wirklichkeit nicht mehr von Phantasie und Beziehungserinnerungen unterschieden werden. Patientin 2 gelingt es am Ende ihrer psychotischen Episode, das Erlebte zu relativieren, Patient 1 hat sich offenbar über diesen Denkmodus in seiner Parallelwelt fixiert.

Patientin 2 gibt uns zunächst Einblick in die Lebenssituation, die den psychotischen Verarbeitungsmodus auslöst: Sie ist in ihrem Liebeswunsch an den Lektor ihrer Diplomarbeit frustriert. Über reale Versuche, ihre Wünsche umzusetzen, erfahren wir nichts, wohl aber, dass sie sich von ihrem realen Partner zunehmend distanziert und an eine Trennung denkt. Nach Abgabe ihrer Diplomarbeit, womit offenbar auch die Kontaktmöglichkeit mit dem Lektor beendet ist, folgt eine Phase des sozialen Rückzugs mit erhöhtem Alkoholkonsum ${ }^{65}$ und Empfindungen zunehmender Depression und innerer Leere, welche die psychische Regression, den Libido-Abzug abbilden. Hier beginnt die manifeste wahnhafte Produktion: Der Restitutionsversuch verarbeitet die Wiederkehr versagter und verdrängter Liebeswünsche auf dem Niveau der Regression, dem „Fixierungsniveau“, das auf die ursprüngliche Versagung in der Kindheit und seine projektive Verarbeitung verweist: Er besteht zunächst in dem Versuch, eine Erklärung für die aktuelle Liebesversagung zu finden, die schon vorher im Verein mit angstbesetzten, an die Erledigung der Diplomarbeit geknüpften Minderwer-

62 Freud hat diesen Begriff als Bezeichnung für die in heutiger Terminologie sogenannte Schizophrenie vorgeschlagen.

63 Demenz $=($ hier $)$ Dementia praecox $=$ Schizophrenie

64 Freud S (1911) Psychoanalytische Bemerkungen über einen autobiographisch beschriebenen Fall von Paranoia. SA VII, S. 199

65 Der Alkohol wird als Spannungslöser eingesetzt, um Affekt- und Triebdruck zu mildern, bis die Patientin damit schlussendlich kein Auslangen findet. Die geschilderte Entzugssituation kann zur Entstehung des psychotischen Zustands beigetragen haben. Der weitere Ablauf und die Wahninhalte sind nicht durch ein alleiniges Entzugsdelir erklärbar. 
tigkeitsvorstellungen gestanden hat. Diese „Erklärung“ erfolgt durch eine Verknüpfung mit der Nazivergangenheit ihres Urgroßvaters. (Wie wir später erfahren, ist der begehrte Mann jüdischer Herkunft.) Der triebhafte Aspekt und die regressive Bewegung vermitteln sich geradezu wörtlich in der Beschreibung des „Dranges in den Keller" und in die Vergangenheit ihrer familiären Geschichte, d.h. zu den Vorstellungen ihres Ursprungs. Der destruktive Vorstellungsaspekt drückt (als Negativabdruck, Ausdruck der Ambivalenz) das Ausmaß der Wut über die Liebesversagung aus, er entspricht dem aggressiven Anteil der Triebmischung, der hier (in projizierter Form abgewehrt) hervortritt.

Der Zusammenhang mit der daran anschließenden Urszenenvorstellung, die als Vergewaltigung der Mutter durch den Vater phantasiert wird, findet sich bereits in den optischen und akustischen Halluzinationen von Leichenbergen und Schreien und Jammern. Sie selbst erlebt sich in der „Vorhölle“, im höllenhaften Vorzimmer zur (Lust-) Hölle, zu der sich das elterliche Schlafzimmer verwandelt, d.h. sie erlebt sich als von der ersehnten Triebbefriedigung ausgeschlossen. Dabei tauchen, auch wegen des damit verbundenen Neides, aggressive Regungen gegen beide Elternteile auf, die mit Größenvorstellungen durchsetzt sind (,als Jahrhundertmord in die Geschichte eingehen“). Gleichzeitig nimmt sie - über die Identifizierung mit der sich bemächtigenden Rolle und über die Aneignung des den Penis symbolisierenden Messers - die Position des Vaters (gegenüber der Mutter) ein. Somit scheint die frühkindliche Mutter als eigentliches Liebesobjekt den Hintergrund zu bilden. Es ist dies die „negative ödipale Situation“, die das Begehren des gleichgeschlechtlichen Elternteils, also die von Freud postulierte „homosexuelle“Wunschregung und ihre Wunscherfüllung, darstellt. (Parallele zu der Schreber'schen Verwandlung in ein „Weib“ in Freuds Fallbericht.)

In der Vorstellung, sich in einen Mann zu verwandeln (in Sartre, der als berühmter Philosoph gleichzeitig die Größenwünsche befriedigt), mit einem Penis, der sich auch dem Freund gegenüber erigiert, mit der Übernahme der „teuflischen Farben“66 (Verdichtung von Teufel und den Farben der Bucheinbände der Sartre-Ausgabe), der fetischistischen Verwendung dieser Farben ${ }^{67}$ in der Kleidung (schwarze Hose, rotes TShirt) und den roten Zetteln mit der Notiz des „vollzogenen Vatermordes“ setzt sich dieser Wunschaspekt, die Rolle des Vaters einzunehmen und seine phallische (Omni-) Potenz zu besitzen, in verschiedenen Bildern fort. (Der Teufel ist ja das Gegenbild Gottes und als Luzifer ${ }^{68}$ der gefallene Lichtengel). Das Elternpaar ergänzt sich über Simone de Beauvoir in Verkörperung der Mutter. Der dramatisierende Charakter, den man auch im späteren Verlauf beobachten kann, weist auf reifere, hysterisch-phallische Persönlichkeitsanteile hin, die sich bei dieser Gelegenheit Ausdruck verschaffen.

Die Identifizierungsfiguren sind aber nicht ausschließlich männlich. Neben dem Ideal Sartre, der nach einem Todes-Wiedergeburts-Erlebnis zum Vorbild ihrer Verwandlungsvorstellung wird, Thomas Bernhard, mit dem sie eine traurige Kindheit, und Oskar Werner, mit dem sie die Alkoholkrankheit verbindet, ermöglicht die Figur der Romy Schneider als Leidensgenossin des Verlustes (der verunglückte Sohn Romy Schneiders hieß David - ein jüdischer Vorname, auch der Geliebte ist jüdischer Herkunft) das Vorbild einer weiblichen Identifizierungslinie. Als weiteres Beispiel für

66 Teufel und Farben als phallisches Symbol

67 Stendhal (1830)

68 Luzifer, lat. Lichtträger, Morgenstern, Venus 
eine weibliche Identifizierungsfigur kann man Elisabeth L. vermuten, die von Maurice Merleau-Ponty ${ }^{69}$ (auf Betreiben ihrer Eltern) verlassene Geliebte, die offenbar infolge ihrer Liebesversagung an einer „sonderbaren Krankheit“ (der Beschreibung nach ein psychotisches Zustandsbild) verstirbt. ${ }^{70}$ Die depressive Gemütslage wird hier projektiv in der Wahl der Personen abgewehrt.

Der Anknüpfungspunkt an die weibliche Rolle erfolgt im Anschluss an die Offenbarung des Vatermordes beim Psychiater, mit dem offenbaren Überleben der Vaterfiguren (des realen Vaters und Freundes, die sie in die psychiatrische Ambulanz gebracht hatten) und dem Annehmen des „Vitamins“ vom Psychiater im Beisein des Vaters (das Medikament als Symbol des Gestilltwerdens). Die Vorstellung, eine Hochzeitsnacht mit dem Mann zu verbringen, dessen Unerreichbarkeit der Ausgangspunkt der Krise gewesen war und der auf unbewusster Ebene die Elternstelle einnimmt - als „Vereinigung der Kinder der Opfer und der Täter“ -, verdeutlicht die Wunscherfüllung und behebt den ödipalen Konflikt des Vater-/Muttermordes. Dazu passt auch die Vorstellung, gleichzeitig weiter mit ihrem Freund liiert zu sein: Jetzt sind wir alle zusammen, alles ist gut, alles ist möglich. (Wir sehen hier auch, wie sich die beiden durch die Regression verdeutlichten Aspekte der Unentschiedenheit - nämlich Unentschiedenheit in der sexuellen Identifizierung und Unentschiedenheit in der Objektwahl - miteinander verbinden.)

Ab hier setzt sich die weibliche Identitätsvorstellung eindeutig fort. Diese ist zunächst geteilt in zwei Figuren, deren kindlich anmutende Gestaltungen im Wechsel auftreten. Die eine Gestaltung mit Romy Schneider als Protagonistin lehnt sich an ein idealisiertes Mutter-Vorbild an: „Wenn ich einmal so groß bin wie du, dann mache ich das, was du dir immer gewünscht hast, für dich“. Hier sind wiederum der narzisstische Beziehungsaspekt und der negative Ödipuskomplex erkennbar, ebenso wie der identifikatorische Beziehungsanteil). Diese Vorstellung erinnert mit phallisch-hysterisch anmutenden Mechanismen an die Zukunftsvorstellungen eines kleinen Mädchens (mit schönen Kleidern herumzustolzieren, einen Film zu drehen, eine Sinfonie zu dirigieren etc.). Die Wahl der Romy Schneider lässt auch weitere assoziative Möglichkeiten zu, etwa die Verknüpfung mit „Sissy“, der Filmrolle, die Romy Schneider berühmt gemacht hat (der Vorname der Patientin lautet Elisabeth, „Sissy“). Prinzessin $\mathrm{zu}$ sein, ist der Traum des kleinen Mädchens. Als Kaiserin aber hat Sissy ein tragisches Leben und auch der frühe Tod von Romy Schneider macht sie zu einer toten Mutter.

Die andere Gestaltung der weiblichen Identitätsvorstellung mit der Figur E.S. (vielleicht mehr an Elisabeth L. ${ }^{71}$ angelehnt) imponiert eher zwanghaft (mit magisch-rituellen Handlungen, Waschzwängen, Mosaike ordnen, in Reimen sprechen) und vertritt mehr die Abwehr- und Sublimierungsbestrebungen, die im Wunsch nach Anerkennung in der Phantasie der Verleihung des „Nobelpreises für die Liebe zur Philosophie“ ihre Entsprechung finden.

Die Vorstellung, mit zwei Männern (welche die Elternfunktion vertreten) zu leben, verschafft sowohl der manifesten heterosexuellen als auch der latent homosexuellen Strebung eine gewisse Geltung, wobei die Vorstellung der Penislosigkeit (Kastration)

69 Wir können annehmen, dass Fr. S. aufgrund ihrer Diplomarbeit mit der Lebensgeschichte M. Merleau-Pontys und mit seinem philosophischen und kulturellen Umfeld wohl vertraut ist.

70 Danzer G (2003)

71 Die tragische Geliebte Merleau-Pontys, die offenbar durch die Verunmöglichung der Beziehung durch ihre Eltern und den Verrat des Geliebten körperlich und psychisch erkrankt und in der Folge verstirbt. 
geleugnet wird (diese Eltern haben beide einen Penis!). Über die Vision der Anerkennung (Nobelpreis) wird die Hoffnung aufrechterhalten, auch einen solchen Phallus zu erlangen. Die Rückkehr in die Realität erfordert Trauerarbeit, um die Versagung zu überwinden.

Im Unterschied zum episodenhaften Wahnablauf bei Patientin 2, der sich wie ein Traumnarrativ unter einem Vergrößerungsglas liest, erscheinen die Wahnbildungen von Patient 1 statisch und wie eine Überlagerung und Verdichtung wiederkehrender Vorstellungsinhalte. Sie kreisen um eine zentrale Thematik: um die Fragen des Geschlechtunterschieds, der Sexualität und der Generationen und der darin vorgenommenen Wertungen von Gut und Böse als Suche nach einem Wertesystem, das der durch Bedrohungen und Kampf geprägten, narzisstischen Welt Sicherheit, Halt und Relativierungsmöglichkeiten entgegensetzen könnte.

Das Wahngebäude von Patient 1 ist durchsetzt von verschiedensten Crößenvorstellungen, die aus dem biblischen Kontext und aus Heldendarstellungen entnommen sind. Wir finden Anteile der Geschichten von Moses, von Jesus, vom Menschenfischer (dem späteren Petrus) und von den Protagonisten des Films „Lohn der Angst“ im Kontext von Todesgefahr, Kampf und Krieg. Aus diesen Elementen werden eine neue Identität und eine neue Biografie gebildet: Der Vater wird durch Hitler ersetzt, wodurch einerseits eine hochgradige Beziehungsambivalenz, Schrecken und Bewunderung ausgedrückt werden und andererseits durch Identifizierung die eigene Bedeutung erhöht wird. Adolf ist der Vorname des Vaters. (Alois) Schicklgruber hieß der Vater Hitlers. Die Abkunft von der Mutter bleibt erhalten - es handelt sich um das „sexuelle“ Stadium des Familienromans. ${ }^{72}$ Die eigene Person wird verdoppelt und vertauscht. Die Mutter ,ist bei den Neonazis“, die von ihm bekämpft werden, womit er sich zum Vollzieher des väterlichen Wunsches macht, die Auswüchse des Naziregimes liquidieren zu lassen. Dieses komplizierte Vorstellungsgefüge ermöglicht es, die eigenen rivalisierenden, aggressiven Impulse sowohl gegenüber den Geschwistern als auch gegenüber deren (und auch seinem?) Vater (als deren Erzeuger) und der Mutter (als deren „Anhängerin“) als väterlichen Auftrag zu rechtfertigen. Gleichzeitig wird damit die homosexuelle Wunschphantasie ausgedrückt, d.h. der Wunsch, durch Unterwerfung unter die Wünsche des Vaters von diesem geliebt zu werden und gleichzeitig identifikatorisch an seiner Macht teilzuhaben .

Herr S. phantasiert sich als auserwählt, die originale Bibel mit den wahren Namen Gottes zu besitzen und zu übersetzen. Sie ist ins Meer gefallen und von Indianern gefunden worden, so wie er selbst. ${ }^{73}$ Diese Bibel wird als machtvolles Instrument beschrieben, dessen Kernstück der Schöpfungsbericht und die Cenealogie, die wahren Namen Gottes, sind, also das Wissen um die Zeugung und um den „wahren“ Vater. Hier bilden sich infantile Sexualforschung und der Wunsch nach dem Wissen und der Macht (des Wissens) des Vaters ab. Aus dieser Bibel, die gleichzeitig die Gemeinsamkeit mit dem (mafiösen) Papst (Vater) darstellt und auch Parteibuch der Antifa ist, bezieht er Kraft. Sie ist Ausdruck der Homoerotik, der Identifizierung mit dem Heldischen und dem Vater, ein phallisches Symbol.

Die Namen der „guten Götter“, Gottvater und Sohn, vermitteln jedoch über ihre „wahren Namen“ das Gegenteil des Guten. Aus Jahwe wird „Jah preisen tut weh“ (schei-

72 Freud S (1909) Der Familienroman der Neurotiker. SA IV, S. 222-226

73 Rank 0 (1908) 
den tut weh), aus Jesus „Komm, rüste uns“ (Komm, Herr Jesus, sei unser Gast und segne, was du uns bescheret hast). Der Sohn rüstet sich gegenüber der Gefahr, die durch den Vater, die Kastrationsvorstellung, die Scheide, die Scheidung verursacht ist. Die Liebes- und Nähewünsche dem Vater gegenüber sind ambivalent, der ödipale Wunsch nach Vatermord wird in Lobpreisung verkehrt.

„Gott Vater ist ein Zwitter, zugleich Mann und Frau, gut und böse“. Noch deutlicher als im Wahngebilde von Patientin 2 sieht man Unschärfe und Vermischung in den Vorstellungen über die Geschlechterdifferenz und der dieser zugesprochenen Eigenschaften, wie sie dem Regressionsniveau entsprechen. Die Erfahrung von Patient 1 ist offenbar, dass man auf Frauen nicht bauen kann („Antichrist = Antigerüst“): Von denen wird man abgeschoben, fallen gelassen; Frauen sind der Grund für Konflikt und Krieg, ihre Sexualität ist verwirrend und unlogisch. Und wie man es dreht und wendet, immer geht es um Sex (die Zahlen 6 und 9). Die als böse und gefährlich erlebten eigenen sexuellen Strebungen werden projiziert und dem Weiblichen zugeordnet - mit den Attributen unlogisch, unkontrolliert, triebhaft, animalisch. Was dabei herauskommt, ist böse: die beim elterlichen Geschlechtsverkehr gezeugten Kinder, das Verlassenheitsgefühl, wenn er sich dabei ausgeschlossen fühlt, und schlussendlich er selbst.

Der gemeinsame Genuss der Droge AOKA, (die Menschen in Tiere verwandelt), die Vereinigung von Frau und Schlange - also der Geschlechtsverkehr - ist die Ursache für das Böse. AOKA ist eine Wortneuschöpfung, die „Koka(in)“ und „k.o.“ (vielleicht auch die „K.O.-Tropfen“ in der Psychiatrie und ihre Verwendungsmöglichkeiten) verdichtet. AOKA ,verleiht übermenschliche Kräfte und macht schizophren“. Es vereint Potenz und Ohnmacht, das Begehren, das Weibliche, den Apfel des Paradieses, die Sexualität, den Sündenfall und die Kastrationsgefahr („,blutige Füße“). Die Polizei hat ein Gegenmittel zu AOKA gefunden (das Gefängnis - seine jetzige Realität im Maßnahmenvollzug -, aus dem er nicht mehr weg will, wo er sich sicher fühlt.) Und er selbst hat gelernt, durch fortwährendes Beten die Wirkung der Medikamente abzuschwächen und die Kugel (seine Liebeswünsche, den mit Liebesenttäuschung zusammenhängenden Schmerz, die Verletzung) in seinem Herzen zu „entmaterialisieren“.

An zwei Stellen gewinnt der merkwürdig unbezogene Erzählton dieser Selbstdarstellung von Herrn S. einen anderen, mehr individuell-emotionalen Charakter. Hier wird ein Zusammenhang mit krankheitsauslösenden Versagungserlebnissen direkter ausgedrückt und es offenbart sich die schmerzhafte persönliche Betroffenheit. Die erste Stelle ist jene, an der er über seine Verletzung spricht: Es handelt sich offenbar um eine psychische Verletzung, denn sie soll im psychiatrischen Krankenhaus behandelt werden (eine Versagungssituation), die „Kugel“, von der er „,in seine Herzspitze getroffen“ wurde und die dort steckengeblieben ist. Er spricht von einem Blutbad, einer riesigen Schlacht zwischen Neonazis und der Antifa. In diesem Zusammenhang beschreibt er Angst, verzerrte Gesichter von Menschen und Beobachtungsgefühle. Hier wird angedeutet, dass sich in diesem Zusammenhang etwas in seinem Leben radikal verändert hat: Er nimmt die Identität seines „Zwillingsbruders“ an, die des schizophren Kranken; die andere Seite ist tot, getötet von der „letzten Frau“, die ihn ,verwechselt“ (= der Mutter, die ihn nicht erkannt, nicht geliebt) hat.

Dies bestätigt eine andere Stelle am Ende des Wahnberichtes: Im letzten Absatz unterscheidet Herr S. schließlich seine „Kriegsschizophrenie“ von seiner „Privatschizophrenie“, welche damit zu tun habe, dass seine echte Mutter nichts von ihm wissen wollte. 
Man muss wohl annehmen, dass im Leben von Herrn S. weder das verankerte Erleben einer Sicherheit vermittelnden, haltenden und bestätigenden Mütterlichkeit noch eine positive männliche Identifikationsmöglichkeit und ein Gefühl des Angenommenseins vonseiten des Vaters ausreichend vorhanden waren und dass das Wahngebäude ein Versuch ist, mit der daraus resultierenden Enttäuschung, Kränkung und Minderwertigkeitsvorstellung zurechtzukommen.

\section{Literatur}

Danzer G (2003) Merleau-Ponty. Kulturverlag Kadmos Berlin Federn P (1978) Ichpsychologie und die Psychosen. Suhrkamp Frankfurt am Main Freud S (1891) Zur Auffassung der Aphasien. Eine kritische Studie. Franz Deuticke Wien Leipzig Freud S (1999) Gesammelte Werke (GW). Fischer Frankfurt am Main

Freud S (1970) Studienausgabe (SA). Fischer Frankfurt am Main

Krejci E (2011) Freuds „Ich-Spaltung im Abwehrvorgang“ und die Erweiterung des Neurosenmodells. Psyche 65, 1 Loch W (2010) Erinnerung, Entwurf und Mut zur Wahrheit im psychoanalytischen Prozess. Gesammelte Schriften. Brandes \& Apsel Frankfurt am Main

May-Tolzmann U (1996) Freuds frühe klinische Theorie (1894-1896). Wiederentdeckung und Rekonstruktion. edition diskord Tübingen

Niederland WG (1978) Der Fall Schreber. Suhrkamp Frankfurt am Main

Rank 0 (1908) Der Mythus von der Geburt des Helden. Wien Turia + Kant

Schreber DP (1903) Denkwürdigkeiten eines Nervenkranken. Kulturverlag Kadmos Berlin

Stendhal (1830) Rouge et Noir. Deutsche Ausgabe Edl E (Hrsg.) (2004) Rot und Schwarz. Carl Hanser München 